\title{
Cisplatin/Temozolomide Regimen
}

National Cancer Institute

\section{Source}

National Cancer Institute. Cisplatin/Temozolomide Regimen. NCI Thesaurus. Code C160531.

A regimen consisting of cisplatin and temozolomide that may be used in the treatment of glioblastoma. 